\title{
LOS FUNDAMENTOS DEL DERECHO DE NECESIDAD EN TIEMPOS DE LA COVID-19
}

\author{
VICENTE ÁLVAREZ GARCÍA \\ Catedrático de Derecho Administrativo \\ Universidad de Extremadura
}

TRC, n. ${ }^{\circ} 48,2021$, pp. 297-314

ISSN 1139-5583

\section{SUMARIO}

I. Introducción. II. Las teorías históricas del derecho de necesidad. III. Un ensayo de reconstrucción dogmática de la teoría de la necesidad.

\section{INTRODUCCIÓN}

Cualquier persona con unos estudios intermedios podría, a buen seguro, enumerar algunos acontecimientos catastróficos que han acontecido en la historia española, europea y universal. Y absolutamente todas podrían indicar, al menos, una: la pandemia de coronavirus en la que nos encontramos inmersos en el tiempo presente.

Muchas de estas catástrofes han sido provocadas por la naturaleza, como los terremotos, las erupciones volcánicas o las grandes inundaciones o incendios. Pero no son las menos, probablemente, las ocasionadas por la mano del hombre, de manera más o menos consciente. Ahí están, como claros ejemplos, los atentados terroristas, los accidentes químicos y nucleares o las sempiternas guerras.

Es cierto que las dimensiones de las catástrofes no han sido siempre las mismas y, consecuentemente, las necesidades de respuesta por los grupos sociales organizados (significativamente los Estados) han sido variables, pero siempre han contado con un protagonista de primer orden, que ha sido el Derecho y, muy en particular, un tipo de Derecho peculiar, como es el Derecho de las Crisis o Derecho de necesidad.

Este Derecho tiene unos caracteres propios que pasan por la necesidad de ofrecer grandes poderes a las autoridades encargadas de hacer frente a la concreta 
catástrofe: para prevenirla, para luchar contra ella cuando irremediablemente ya se ha desencadenado y para reparar de la manera más rápida posible sus consecuencias más atroces. Estos poderes extraordinarios, que naturalmente no están en manos de las autoridades en tiempos de normalidad, son indispensables para hacer frente a las crisis. Sin ellos, sin estos poderes excepcionales no es posible una lucha eficaz contra las diferentes categorías de catástrofes, por lo que las autoridades deben disponer de ellos necesariamente. Pero la historia también enseña que estas autoridades han abusado muy a menudo de los mismos, y no precisamente en beneficio de la comunidad, sino de ellas mismas. El Derecho de necesidad debe lograr un equilibrio entre, por un lado, la atribución de un poder de actuación suficiente para batallar de manera eficaz contra los peligros que se ciernen sobre el grupo social y, por otro, la prevención del riesgo potencial de abuso del mismo.

En este contexto subyacente, el Derecho de necesidad sustituye temporalmente a la legalidad ordinaria, al Derecho de los tiempos de normalidad. Creo que no hay nadie que haya expresado tan bien esta idea como Montesquieu, cuando decía: «Hay casos en los que es necesario correr, por un momento, un velo sobre la libertad del mismo modo que se ocultan las estatuas de los dioses» ${ }^{1}$.

Esta prevalencia temporal del Derecho extraordinario sobre la legalidad ordinaria se justifica en que esta última no es suficiente para la lucha contra las crisis. Durante un tiempo, el que dure estas crisis, el Derecho habilita para tomar las medidas necesarias para superar la adversidad que amenaza al grupo social, pero este fin no justifica todos los medios, sino tan sólo aquellos que son efectivamente necesarios y proporcionados para asegurar la salvaguarda de los fines de interés general (en particular, del más importante de todos ellos: la supervivencia del grupo organizado) frente a los peligros que les amenazan.

Periódicamente se repiten las crisis de gran calado, pero parece cierto que el mundo occidental hacía tiempo que no vivía una catástrofe como la que ahora nos acecha. Es cierto que la historia de la humanidad está llena de terribles pandemias que han diezmado el planeta desde tiempos mitológicos ${ }^{2}$ hasta el pasado siglo (piénsese en la Peste de Atenas, en la Peste Antonina, en la Plaga de Justiniano, en la Gran Peste Negra, en la mal llamada «Gripe Española», etc.), pero ahora nos toca vivir una, que, además, y con toda probabilidad, no será ni siquiera la última ${ }^{3}$.

1 «Il y a des cas où il faut mettre, pour un moment, un voile sur la liberté comme l'on cache les statues des dieux», en De l'esprit des lois, libro XII, capítulo XIX, Ed. Flammarion, 1979, p. 345.

2 Canta la Ilíada en sus primeros versos que las flechas inmortales de Apolo sirvieron para arrojar su cólera mortal contra el ejército griego que tiempo más tarde conquistaría la ciudad de Troya. Cuenta Homero que la cólera divina vino provocada por la afrenta que el rey Agamenón habría causado al sacerdote Crises, al no querer devolverle a su amada hija Criseida, a pesar del rico rescate ofrecido por el padre. La ira del Dios sólo se vio aplacada, y con ello la peste que asolaba a los helenos, con la devolución de la hija al padre, protegido de Apolo, en una embarcación pilotada por el ingenioso Ulises.

3 Como breve introducción a las enseñanzas comunes que pueden extraerse de las grandes epidemias a lo largo de la historia, puede verse Álvarez García, V., Arias Aparicio, F., y Hernández Diez, E., Lecciones jurídicas para la lucha contra una epidemia, Iustel, 2020, pp. 17 y ss. 
El surgimiento de la pandemia del coronavirus ha justificado que de nuevo el Derecho de necesidad haya cobrado un gran protagonismo ${ }^{4}$, por lo que vale la pena recordar cuáles son sus componentes fundamentales. Estudié en diversas universidades francesas, alemanas, belgas y españolas esta cuestión durante el primer lustro de los años noventa del pasado siglo, dando como resultado mis investigaciones un libro que titulé El concepto de necesidad en Derecho Público (Civitas, 1996). Sus conclusiones más de veinticinco años después siguen plenamente vigentes, como podrá comprobar el lector si continúa leyendo las siguientes páginas.

\section{LAS TEORÍAS HISTÓRICAS DEL DERECHO DE NECESIDAD}

\section{Una idea preliminar}

La necesidad es un concepto que desde siempre ha tenido extraordinarias repercusiones en el mundo del Derecho en general, y del Derecho público en particular ${ }^{5}$.

En su configuración tradicional, la necesidad se concibe como un fenómeno que faculta (o, incluso, que obliga) a los Poderes Públicos para excepcionar el Derecho existente en un momento dado, y para adoptar las medidas indispensables en orden a la salvaguarda de los bienes esenciales para la vida de la comunidad (y, muy en particular, la propia subsistencia del Estado en su concepción más clásica), cuando éstos fuesen puestos en peligro por la concurrencia de una situación de «extraordinaria gravedad», y siempre y cuando, además, el Derecho preexistente fuese inadecuado o insuficiente para superar la situación de peligro.

\section{La distinción tradicional entre los conceptos de necesidad y de urgencia}

Junto al concepto de necesidad, la doctrina tradicional ha elaborado el de urgencia, diferenciándose ambas figuras en base al elemento fáctico (en concreto, en base a la mayor o menor gravedad de la coyuntura $)^{6}$.

4 Sobre la abundantísima literatura jurídica que ha ido apareciendo desde la adopción de las primeras medidas sanitarias para hacer frente al coronavirus allá por el mes de marzo del año 2020, véase el extenso índice bibliográfico contenido en este número monográfico.

5 Y es que, como decía J. LAmARQue, «la idea de la necesidad es de todas las épocas y de todos los regímenes» («La théorie de la nécessité et l'article 16 de la Constitution de 1958», RDP, 1961, p. 596).

6 Sobre esta forma de construir el concepto de urgencia, pueden destacarse el clásico trabajo de CLAVERo ArÉvalo, M.F., «Ensayo de una teoría de la urgencia en el Derecho administrativo», RAP, n. ${ }^{\circ} 10$, 1953; y otros dos de GABOLDE, Ch., Essai sur la notion d'urgence en droit administratif français (Les pouvoirs d'urgence de l'Administration), Tesis, Universidad de Paris, 1951, y «L'exception d'urgence en droit administratif», Dalloz, 1952. 
Mientras que la necesidad exigiría para poder desplegar sus efectos la concurrencia de una situación de extrema gravedad (normalmente poco frecuentes en la vida del Estado) que pusiese en peligro un fin esencial de la comunidad, la urgencia tan sólo entraría en juego ante situaciones menos graves, menos angustiosas (y, por tanto, más frecuentes para la comunidad).

Las diferencias en la conceptualización de la necesidad y de la urgencia encontrarían su proyección práctica en la diversidad de efectos de una y otra figura.

A) La necesidad tendría unas consecuencias mucho más drásticas, mucho más enérgicas, justificando incluso la alteración temporal del principio de la división de poderes.

B) La urgencia, sin embargo, tendría unos efectos mucho más modestos que la necesidad, permitiendo únicamente pequeñas alteraciones en las reglas jurídicas, procedimentales o competenciales (pero, en este último caso, siempre dentro del mismo Poder del Estado).

\section{Las construcciones dogmáticas del concepto de necesidad en la historia}

Si bien ha existido tradicionalmente unanimidad en la doctrina acerca de la consideración de la necesidad como elemento legitimador de las actuaciones de los Poderes Públicos contrarias al Derecho ordinario, cuando tales actuaciones son indispensables para superar las circunstancias excepcionales que amenazan un fin esencial, no puede decirse que ocurra lo mismo respecto de la fundamentación teórica y del valor jurídico atribuido a nuestro concepto.

Han existido a lo largo de la historia, en efecto, dos teorías doctrinales bien diferentes en torno a la naturaleza de la necesidad:

A) Para la teoría fáctica de la necesidad, cuyos orígenes inmediatos se encuentran en la teoría clásica alemana del Notrecht, serían las circunstancias fácticas, y no las normas jurídicas, las que justificarían las actuaciones de necesidad de los Poderes Públicos. Estas actuaciones serían siempre contrarias a Derecho, pero su ilegalidad resultaría excusada en base a una situación de extraordinario peligro, que exigiría una actuación exorbitante de los Poderes Públicos ${ }^{7}$.

B) Para la teoría jurídica de la necesidad, ésta sería un fenómeno jurídico, con un fundamento y unos efectos de esta naturaleza. Pero, no obstante, si

7 Toda esta teoría puede resumirse en la frase que da título a una obra de J. KöHLER, Not kennt kein Gebot, y que no es sino traducción al mundo jurídico de la filosofía hegeliana sobre las relaciones entre el Estado y el Derecho. El título completo de la obra de KöHLER es Not kennt kein Gebot. Die Theorie des Notrechts und die Ereignisse unserer Zeit, Verlagsbuchhandlung Dr. Rothschild, Berlin und Leipzig, 1915. 
todas las doctrinas que se incardinan dentro de esta teoría encuentran la justificación de la necesidad dentro del propio Derecho, no todas lo hacen del mismo modo: algunas se contentan con buscar el fundamento jurídico inmediato a la necesidad (como la teoría de la voluntad presunta del Constituyente o del Legislador...), mientras que otras, más ambiciosas, intentan encontrar el fundamento último de la necesidad (la teoría de la legítima defensa del Estado, la de la continuidad de los servicios públicos...).

\section{Las plasmaciones prácticas del modelo teórico del concepto de necesidad}

El modelo teórico de la necesidad ha encontrado diferentes plasmaciones prácticas a lo largo de la historia.

A) La justificación jurisprudencial de los poderes de necesidad encuentra su máxima expresión en la «jurisprudencia francesa de las circunstancias excepcionales». El Consejo de Estado galo ha efectuado desde comienzos del siglo XX un riguroso control a posteriori de las medidas de necesidad dictadas por los Poderes Públicos. El conjunto de sus diferentes decisiones ha llegado a constituirse con el paso del tiempo en un auténtico Derecho jurisprudencial de necesidad ${ }^{8}$.

B) La técnica anglosajona de la exoneración parlamentaria (o del bill of indemnity). Aquí, el Parlamento procede a una sanación ex post facto de las actuaciones ilegales de los Poderes Públicos adoptadas en situaciones de crisis 9 .

C) La previsión apriorística por el Derecho positivo de los poderes de necesidad: han sido innumerables los intentos del Constituyente y del Legislador de prever positivamente un Derecho de excepción o, más ampliamente, de necesidad. Existen, a este respecto, dos técnicas básicas extremas entre las cuales pueden existir múltiples variantes. A saber:

a) La primera de ellas (cuyo modelo clásico era el «estado excepcional» ${ }^{10}$ ) obedece a la idea de tipificar taxativamente (en base a consideraciones

8 Entre la abundante bibliografía francesa sobre esta doctrina jurisprudencial, puede consutarse Fahmy-Medany, M., La théorie des circonstances exceptionnelles en droit administratif français et égyptien, Tesis, Université de Paris, 1954; Mathiot, A., «La théorie des circonstances exceptionnelles», en L'évolution du Droit Publique, Etudes offets à A. Mestre, Sirey, 1956; y NizARD, L., La jurisprudence administrative des circonstances exceptionnelles et la legalité, LGDJ, 1962.

9 Sobre esta cuestión, pueden verse, por todas, las obras clásicas de DiCEY, D.V., Introduction to the study of the Law of the Constitution, London, 1908; y de Schwartz, B., Los Poderes del Gobierno, 2 vols., Universidad Nacional Autónoma de México, 1966.

10 Sobre esta técnica de los estados excepcionales durante el constitucionalismo español son sencillamente excelentes los libros de Ballbé, M., Orden público y militarismo en la España constitucional (1812-1983) y de Cruz Villalón, P., El estado de sitio y la Constitución, Madrid, 1980. 
cualitativas o cuantitativas), mediante el establecimiento de «cláusulas estándar», una serie de supuestos de hecho que harían peligrar un fin de interés general, ligándoles específicas consecuencias jurídicas ${ }^{11}$.

b) La segunda de las variantes (cuyo modelo paradigmático es la «dictadura constitucional» ${ }^{12}$ ) consiste en recurrir al establecimiento de «cláusulas generales de habilitación», mediante las cuales se faculta a una determinada autoridad pública para que adopte las «medidas necesarias» para superar exitosamente todas aquellas situaciones extraordinarias que se puedan presentar en la vida cotidiana del Estado, y frente a las que el ordenamiento positivo no ha establecido ninguna respuesta jurídica concreta o, habiéndolo hecho, ésta resulta insuficiente o inadecuada.

\section{UN ENSAYO DE RECONSTRUCCIÓN DOGMÁTICA DE LA TEORÍA DE LA NECESIDAD}

\section{Consideraciones introductorias: la necesidad no constriñe su virtualidad a tan sólo los peligros extremos para la vida del Estado}

Frente a las concepciones tradicionales de la necesidad, y desde una perspectiva renovadora, puede constatarse cómo nuestro concepto no constriñe su virtualidad exclusivamente a las situaciones de crisis extraordinarias para la vida del Estado, sino que, por el contrario, su contenido es mucho más amplio, pudiendo desplegar sus efectos en todo tipo de situaciones, incluidas, claro está, las de normalidad generalizada.

11 Este es el modelo seguido tradicionalmente por el Derecho español. En la actualidad, el art. 116 CE prevé la existencia de tres estados excepcionales: el estado de alarma, el estado de excepción y el estado de sitio. Es cierto que nuestra Carta Magna no tipifica directamente ni en qué supuestos fácticos es posible la declaración de alguno de ellos, ni cuáles serán las medidas de necesidad que podrán ser adoptadas durante su vigencia (salvo en lo que se refiere a la suspensión de derechos fundamentales prevista por el art. 55.1 CE). Estas tareas se han cumplimentado en la L.O. 4/1981, de 1 de junio, de los estados de alarma, excepción y sitio (LOAES). Partiendo de esta idea, y a diferencia de lo que ha pasado a lo largo de nuestra historia constitucional, el legislador hispano, siguiendo la experiencia de la Ley Fundamental de Bonn, no optó, a la hora de elaborar la LOAES, por una regulación gradualista de los estados de emergencia, de tal manera que en función de la gravedad del peligro se acudiese a uno o a otro de mayor nivel de severidad, sino que se siguió un criterio cualitativo: se emplearía un estado u otro en función del tipo de peligro, de forma tal que para la lucha contra las catástrofes se recurriría al estado de alarma, si estuviese en juego el orden público por causas interiores se acudiría al estado de excepción y, sólo, y aquí sí entraría una cierta dimensión gradualista, para los peligros extremos de naturaleza insurreccional interna o de amenaza exterior contra la unidad del Estado o para el orden constitucional podría activarse el estado de sitio (Álvarez García, V., Arias Aparicio, F., y Hernández DiEz, E., Lecciones jurídicas para la lucha contra una epidemia, Iustel, 2020, p. 96).

12 Probablemente el mayor experto desde una perspectiva jurídica sobre esta técnica haya sido C. SCHMITT, que la explicó de manera extraordinariamente brillante en su obra La dictadura, Alianza Universidad, 1985. 
La necesidad, pues, opera en todo tipo de circunstancias como instrumento jurídico de conexión entre un elemento circunstancial o fáctico (los peligros, las crisis, las catástrofes) y el elemento teleológico, integrado por el conjunto de fines esenciales para el grupo social en un lugar y un tiempo determinados, y cuya protección y realización tienen encomendada los Poderes Públicos.

La necesidad así entendida puede operar de dos formas:

A) En primer lugar, mediante su traducción o proyección en normas positivas del más diverso rango (constitucional, legal o reglamentario) ${ }^{13}$.

B) En segundo lugar, como principio general del Derecho, integrador del ordenamiento, que sirve de base jurídica o de causa de justificación de las medidas que indefectiblemente los Poderes Públicos se viesen obligados a adoptar praeter legem o, incluso, contra legem, para lograr la realización de un determinado fin esencial para la comunidad en unas circunstancias fácticas concretas que lo hicieran peligrar.

\section{La obligación de los poderes públicos de asegurar la realización de los fines esenciales de la comunidad}

Todo grupo social mínimamente organizado persigue una serie de objetivos o de fines orientados a asegurar, en primer lugar, su propia supervivencia y, en segundo lugar, su correcto funcionamiento.

Para la realización de estas funciones la comunidad se dota de unos Poderes Públicos, siendo precisamente la necesidad de llevar a cabo esta tarea lo que justifica su creación y su pervivencia. Los poderes públicos, por tanto, no están tan

13 El art. 116 CE (desarrollado por la LOAES) recoge el Derecho constitucional de excepción (con los estados de alarma, de excepción y de sitio), pero no son éstas las únicas normas de necesidad existentes en nuestro país. En efecto, y por un lado, aunque el ordenamiento jurídico hispano carece de una ley sanitaria específica para la lucha contra las epidemias (a diferencia de lo que, por ejemplo, sucede en otro Estado descentralizado como Alemania con la Infektionsschutzgesetz del año 2000), sí que tiene diversas disposiciones para hacer frente a las crisis de salud pública, como el art. 26 de la Ley 14/1986, de 25 de abril, General de Sanidad; el art. 54 de la Ley 33/2011, de 4 de octubre, General de Salud Pública; o la tan breve como importante L.O. 3/1986, de 14 de abril, de Medidas Especiales en Materia de Salud Pública. El art. 3 de esta última norma sanitaria, por ejemplo, contiene una cláusula general sanitaria que permite adoptar las «medidas necesarias» para hacer frente a una enfermedad infecciosa: «Con el fin de controlar las enfermedades transmisibles — dice este precepto_- la autoridad sanitaria, además de realizar las acciones preventivas generales, podrá adoptar las medidas oportunas para el control de los enfermos, de las personas que estén o hayan estado en contacto con los mismos y del medio ambiente inmediato, así como las que se consideren necesarias en caso de riesgo de carácter transmisible». Por otro lado, existe otra clase de normas legales de necesidad que sirven para la lucha frente a todo tipo de emergencias, como sucede con la Ley 17/2015, de 9 de julio, del Sistema Nacional de Protección Civil (que, para hacer frente a las catástrofes más extremas, prevé la técnica de las «emergencias de interés nacional»), y con la Ley 36/2015, de 28 de septiembre, de Seguridad Nacional (que atribuye unos amplísimos poderes de necesidad al Presidente del Gobierno mediante la instauración de la figura de la «situación de interés para la seguridad nacional»). 
sólo facultados, sino que están también obligados al cumplimiento de esta misión ${ }^{14}$. Y, en la medida en que esto es así, resulta imprescindible dotarles de los medios suficientes para ello, puesto que no tendría el más mínimo sentido crear unos órganos, encargarles unas funciones, e impedirles, a renglón seguido, su realización por la falta de medios.

Entre estos medios, y en lo que a nosotros atañe, se encuentra el Derecho, que no constituye, por tanto, un fin en sí mismo.

A veces los medios jurídicos están positivizados, en cuyo caso los poderes públicos pueden acudir a ellos para el desempeño de su función de asegurar la realización de un fin esencial, superando la situación de peligro que le amenaza, sin tener que buscar ninguna otra justificación suplementaria para su actuación. Pero no siempre ocurre así. No siempre disponen los Poderes Públicos de específicos medios jurídicos positivizados para la realización de su misión. En estos casos, el medio jurídico (y, por tanto, la justificación) para su actuación vendrá directamente del principio general de necesidad (cuyo juego estaría permitido por el art. 1.4 $\mathrm{CC}^{15} \mathrm{y}$, sobre todo, por el art. $103.1 \mathrm{CE}^{16}$ ) que operaría como norma de habilitación, a la vez que de limitación, para una concreta actuación de los Poderes Públicos ante una determinada situación de peligro para un fin esencial de la comunidad.

\section{Los elementos configuradores del concepto de necesidad}

La necesidad es un concepto jurídico indeterminado, cuya estructura interna está conformada por la presencia de dos tipos de componentes o de elementos: uno teleológico y fáctico el otro.

A) El elemento finalista o teleológico está integrado por los fines u objetivos que son esenciales para la comunidad.

La necesidad es, en efecto, un concepto con un marcado carácter teleológico, definiéndose en cada caso concreto en función del fin específico que los Poderes

14 La obligación de los poderes públicos de realizar las tareas que tienen encomendadas (y, en particular, asegurar la supervivencia ordenada del Estado) les diferencia de manera esencial de los sujetos privados. Cualquier ciudadano puede decidir si, en caso de necesidad, recurre o no a la legítima defensa. Los poderes públicos de un Estado no tienen esa opción: en caso de peligro para su existencia o para su correcto funcionamiento deben, a priori, y como regla general, adoptar las «medidas necesarias» para su defensa. Sobre esta cuestión, véase más ampliamente Álvarez García, V., «Las facultades de intervención de las Fuerzas Armadas ante las situaciones de crisis», en Álvarez García, V., y Moradiellos García, E., Las Fuerzas Armadas ante las situaciones de crisis, Iustel, 2020, pp. 73 y ss.

15 Este precepto prevé que: «Los principios generales del derecho se aplicarán en defecto de ley o costumbre, sin perjuicio de su carácter informador del ordenamiento jurídico».

16 Este precepto dispone, en lo que ahora nos interesa, que: «La Administración Pública sirve con objetividad los intereses generales (...), con sometimiento pleno a la ley y al Derecho». 
Públicos tienen la obligación de realizar en un momento dado. La existencia de unos fines comunitarios esenciales justifica, tal y como se ha señalado, la creación en el seno de la comunidad de unos Poderes Públicos destinados a asegurar su realización, debiendo ser el Derecho un instrumento destinado a permitir el desempeño correcto de tal tarea. Esto no significa que el fin justifique todos los medios. El fin tan sólo justifica la utilización de aquellos medios que son necesarios y proporcionados (entendiendo este último concepto en sentido amplio) para su realización.

Los fines u objetivos cuya realización permite el recurso a los poderes de necesidad son, en principio, aquellos que constituyen un «mínimo vital», en un momento y en un espacio geográfico determinado, para un grupo social mínimamente organizado.

El primero de los fines constitutivos de ese mínimo vital es la continuidad de la vida del propio grupo social (Estado): su pura y simple supervivencia.

Ligados a este fin verdaderamente primario, existen otros considerados también como esenciales tanto por la doctrina, como por la jurisprudencia y los textos positivos: en primer lugar, el mantenimiento de los servicios esenciales para el funcionamiento de la comunidad; y, en segundo lugar, el mantenimiento del orden público o, lo que es lo mismo, el mantenimiento de unos niveles mínimos en la seguridad, en la salud y en la tranquilidad pública, niveles mínimos necesarios para asegurar una convivencia ciudadana pacífica.

Junto a estos fines con una dimensión comunitaria innegable (sin que ello signifique que no la tengan también individual), en la actualidad, doctrina y jurisprudencia admiten la existencia de otros fines (como la garantía de los derechos y libertades de los ciudadanos, el salvamento de personas y cosas...) con una marcada (aunque no exclusiva) dimensión individual, que justifican, también, el recurso a los poderes de necesidad.

B) El elemento circunstancial o fáctico es el segundo de los componentes indispensables en la construcción del concepto de necesidad.

La necesidad exige, en efecto, la presencia de unas circunstancias de hecho, de una situación de peligro o de potencial amenaza (real, actual y con un alcance geográfico determinado) para alguno de los fines reconocidos como esenciales por el grupo social, amenaza a la que tienen que hacer frente de manera obligada los Poderes Públicos a través de sus potestades de necesidad.

A diferencia de lo que ocurre con el elemento teleológico, en el que los fines que lo integran pueden ser tipificados de una manera apriorística, las situaciones de hecho que potencialmente pueden integrar el elemento fáctico son infinitas, y, por tanto, cualquier intento de determinación previa por los Poderes Públicos estaría abocado al fracaso. Esta indeterminación apriorística, esencial al elemento fáctico, hace consecuentemente imposible la previsión de una respuesta normativa expresa para conectar todos y cada uno de los posibles binomios fáctico-teleológicos que podrían presentarse en la vida del grupo social. En los casos en los 
que no exista tal previsión normativa es en los que la necesidad, en tanto que principio general del Derecho, tendrá un campo especialmente abonado para desplegar su virtualidad.

El contenido específico del elemento fáctico, de la situación de peligro, puede verse enormemente influenciado, y esto es probablemente una de las causas fundamentales de su indeterminación, por factores de naturaleza temporal (mayor o menor inminencia — del peligro-) o cualitativa (mayor o menor gravedad — del peligro-). La influencia de estos dos tipos de factores en la configuración del peligro tiene una repercusión directa sobre el concepto de necesidad, dando lugar a la aparición de los conceptos de urgente necesidad (o urgencia), extraordinaria necesidad y extraordinaria y urgente necesidad.

a) La urgencia no es, como se ha sostenido tradicionalmente, un concepto autónomo con respecto a la necesidad, con una operatividad estrictamente limitada a hacer frente a las situaciones de peligro menos graves o más banales que se puedan presentar en la vida de la comunidad. La urgencia es, bien al contrario, una categoría específica de la necesidad: la necesidad de actuar con rapidez ante la inminencia de la amenaza de materialización de la situación de peligro (urgencia preventiva) o, una vez que éste ya se ha materializado, para reparar con la mayor celeridad posible los daños ya producidos (urgencia reparatoria o reparadora).

b) El concepto de necesidad puede venir modulado, en segundo lugar, por factores cualitativos, es decir, por la mayor o menor gravedad que revista la situación de peligro en relación al fin. Cuando la gravedad de la situación de peligro sobre el fin esencial es extrema, surge la extraordinaria necesidad que habilita a los Poderes Públicos para la adopción de una respuesta muy drástica, en contraste con aquellos otros supuestos en los que el peligro es tremendamente leve, donde la necesidad simple justificará tan sólo una respuesta de los Poderes Públicos de muy corto alcance.

c) La extraordinaria y urgente necesidad viene definida por la concurrencia simultánea del factor temporal y del cualitativo (gravedad de la situación). En efecto, cuando la situación de peligro alcance las cotas de máxima inminencia y extrema gravedad, aparecerá el concepto de extraordinaria y urgente necesidad, concepto que justificará las máximas alteraciones en las reglas jurídicas que disciplinan ordinariamente la actuación de los Poderes Públicos.

\section{Los efectos fundamentales del concepto de necesidad}

La necesidad no es tan sólo un concepto teórico, sino que, por el contrario, tiene unas consecuencias prácticas verdaderamente impresionantes. La necesidad, en efecto, tiene dos tipos de efectos fundamentales: uno, negativo; y otro, positivo. 
A) Centrándome en el primero de ellos, hay que decir que la necesidad permite excepcionar temporalmente la aplicación del Derecho «normal» u «ordinario». La necesidad habilita, ciertamente, para remover todos los obstáculos jurídicos a la actuación de los Poderes Públicos cuando ello sea indispensable para la realización de un fin esencial para la comunidad.

B) Este efecto negativo de inaplicación del Derecho se ve completado con otro positivo: la necesidad faculta a los Poderes Públicos para adoptar la regla concreta, la medida jurídica precisa, que, ante un determinado binomio fáctico-teleológico, permitirá realizar el fin, superando la situación de peligro. De esta manera, la necesidad se erige en una técnica de adaptación del Derecho a la realidad social.

Ambos efectos de la necesidad, el negativo o derogatorio (temporalmente hablando), y el positivo o adaptador, encuentran una concreta proyección sobre las distintas reglas jurídicas que disciplinan la actuación ordinaria de los Poderes Públicos, permitiendo su alteración ${ }^{17}$. La necesidad justifica, en efecto, una alteración temporal de:

a) Las reglas de competencia.

La necesidad permite un reajuste competencial dentro de una misma Administración Territorial, o entre diferentes Administraciones Territoriales, o, incluso, puede llegar a justificar las intervenciones extraordinarias de simples particulares en funciones propias de los Poderes Públicos.

Un ejemplo magnífico de esta modificación de las reglas de competencia lo encontramos en nuestro Derecho constitucional de excepción, como hemos tenido ocasión de comprobar con la declaración del estado de alarma para hacer frente a la primera ola de la COVID-19 mediante el Real Decreto 463/2020, de 14 de marzo. Su art. 4 sirve, en efecto, para centralizar en manos del Gobierno de la Nación (siguiendo las previsiones del art. 7 LOAES) las competencias para la

17 El Tribunal Constitucional ha ratificado esta idea de que los efectos jurídicos por excelencia del Derecho constitucional de excepción consisten en la sustitución temporal de los poderes ordinarios de los poderes públicos por poderes de necesidad: «(...) todos los estados que cabe denominar de emergencia ex art. 116 CE y también por tanto, el de menor intensidad de entre ellos, esto es, el de alarma, suponen, como es evidente y así resulta de su regulación en la Ley Orgánica 4/1981, de 4 de junio, de los estados de alarma, excepción y sitio, excepciones o modificaciones pro tempore en la aplicabilidad de determinadas normas del ordenamiento vigente, incluidas, en lo que ahora importa, determinadas disposiciones legales, que sin ser derogadas o modificadas sí pueden ver alterada su aplicabilidad ordinaria (arts. 9 a $12 ; 16$ a 30; 32 a 36 de la Ley Orgánica 4/1981, de 4 de junio, de los estados de alarma, excepción y sitio), pues el fundamento de la declaración de cualquiera de estos estados es siempre la imposibilidad en que se encuentran las autoridades competentes para mantener mediante 'los poderes ordinarios' la normalidad ante la emergencia de determinadas circunstancias extraordinarias (art. 1.1 de la Ley Orgánica 4/1981, de 4 de junio, de los estados de alarma, excepción y sitio)» (ATC 7/2012, de 13 de enero, FJ 4; y STC 83/2016, de 28 de abril, FJ 9). 
lucha contra la pandemia. En este sentido, y a título meramente ilustrativo, las competencias sanitarias o las policiales pasan temporalmente a manos estatales ${ }^{18}$.

\section{b) Las reglas de procedimiento.}

La necesidad puede repercutir sobre el desarrollo del conjunto de trámites que conforman el procedimiento administrativo en circunstancias ordinarias con dos propósitos principales: ya sea, por un lado, el facilitar la adopción de decisiones o de actuaciones por parte de los Poderes Públicos, ya sea, por otro, el garantizar la seguridad jurídica y no perjudicar los derechos e intereses legítimos de los ciudadanos.

En este contexto, la necesidad puede permitir, en primer lugar, la simplificación del procedimiento ordinario (mediante la supresión de ciertos trámites o la reducción de plazos); en segundo lugar, la sustitución del procedimiento ordinario por otro de urgencia (reduciendo a la mitad los plazos establecidos para el procedimiento ordinario); en tercer lugar, la supresión total de todo procedimiento (cuando, por ejemplo, se ejerce la coacción administrativa directa o se acude a la contratación de emergencia); y, en cuarto lugar, la suspensión de los procedimientos (mediante la paralización del cómputo de los plazos administrativos).

Son múltiples, con este trasfondo, los ejemplos de modulaciones de las reglas procedimentales que se han producido desde la declaración del estado de alarma el 14 de marzo de 2020. Baste señalar el recurso a las requisas de bienes (que, como es sabido, son expropiaciones exprés — sin seguir realmente ningún procedimiento administrativo- - o a la suspensión de los plazos administrativos, medidas ambas previstas por el RD 463/2020, de 14 de marzo. En efecto, las requisas, con la base jurídica ofrecida por el art. 11 b) LOAES, están contempladas en el art. 8.1 de dicho Real Decreto de necesidad, mientras que las suspensiones de plazos procesales, de plazos administrativos y de plazos de prescripción y caducidad están previstas en las disposiciones segunda a cuarta de esta norma de emergencia ${ }^{19}$.

18 Desde una perspectiva jurisprudencial, nuestro Tribunal Constitucional ha sancionado la juridicidad de este tipo de medidas recentralizadoras durante la vigencia del estado de alarma en su pronunciamiento con motivo de la «crisis de los controladores aéreos» de diciembre de 2010. En efecto, dice el Alto Tribunal —parafraseando el contenido de varios preceptos de la LOAES — que: «(...) la autoridad competente es el Gobierno o, por delegación de éste, el Presidente de la Comunidad Autónoma cuando la declaración afecte exclusivamente a todo o parte de su territorio. Quedan bajo las órdenes directas de la autoridad competente todas las autoridades civiles de la Administración pública del territorio afectado por la declaración, los integrantes de los cuerpos de policía de las Comunidades Autónomas y de las corporaciones locales, así como los demás trabajadores y funcionarios de las mismas, pudiendo imponérseles servicios extraordinarios por su duración o por su naturaleza. Asimismo, los funcionarios y las autoridades en caso de incumplimiento o resistencia a las órdenes de la autoridad competente pueden ser suspendidos de inmediato en el ejercicio de sus cargos, pudiendo asumir también la autoridad competente las facultades de las autoridades que hubiesen incurrido en aquellas conductas cuando fuera necesario para el cumplimiento de las medidas acordadas en ejecución de la declaración del estado de alarma (arts. 7, 9 y 10 de la L.O. 4/1981)» (STC 83/2016, de 28 de abril, FJ 8).

19 Sobre esta cuestión, véase Álvarez García, V., y Arias Aparicio, F., «La incidencia del Derecho de necesidad en el procedimiento administrativo: la alteración de las reglas procedimentales por motivos de necesidad», Revista General de Derecho Administrativo, n. ${ }^{\circ}$ 54, 2020. 
c) Las reglas de forma.

Piénsese, a este respecto, en el siguiente ejemplo tomado de nuestro vigente Derecho constitucional de emergencia. La declaración y la prórroga del estado de alarma (así como la declaración y la prórroga del estado de excepción) corresponden al Gobierno de la Nación mediante una disposición que adoptará la forma jurídica de Real Decreto (art. 116.2 CE). Debe tenerse en cuenta que, como regla general, el Real Decreto es la forma jurídica que revisten habitualmente las disposiciones generales y singulares adoptadas por el Consejo de Ministros. Esta forma jurídica (a diferencia de las de Real Decreto-ley o de Real Decreto Legislativo) sirve para indicar que nos encontramos ante actuaciones de rango inferior a la ley (esto es, de valor reglamentario) [art. 24.1 c) de la Ley 50/1997, de 27 de noviembre, del Gobierno]. No obstante, como excepción, y según nuestra jurisprudencia constitucional, los Reales Decretos de declaración ${ }^{20}$ y de prórroga ${ }^{21}$ del estado de alarma no poseen un simple rango reglamentario, sino que disponen de fuerza de $l e y^{22}$.

\section{d) Las reglas de contenido.}

La regulación existente en nuestro Derecho constitucional de los estados de emergencia ofrece magníficos ejemplos de modulaciones (más allá de las limitaciones y de las restricciones admitidas para el caso del estado de alarma ${ }^{23}$ ) de las reglas jurídicas materiales o de contenido en situaciones de necesidad. Así, por

20 En relación con el Real Decreto de declaración del estado de alarma, dice nuestro Tribunal Constitucional que: «(...) aunque formalizada mediante decreto del Consejo de Ministros, la decisión de declarar el estado de alarma, dado su contenido normativo y efectos jurídicos, debe entenderse que queda configurada en nuestro ordenamiento como una decisión o disposición con rango o valor de ley. Y, en consecuencia, queda revestida de un valor normativo equiparable, por su contenido y efectos, al de las leyes y normas asimilables cuya aplicación puede excepcionar, suspender o modificar durante el estado de alarma» (STC 83/2016, de 28 de abril, FJ 10).

21 Si el Real Decreto declaratorio del estado de alarma tenía, a pesar de adoptar esta forma jurídica, rango o fuerza de ley, este mismo valor jurídico posee la prórroga gubernamental del estado de alarma, que se dicta con esta misma forma, tras la autorización expresa del Congreso de los Diputados. Dice, a este respecto, el Tribunal Constitucional que: «(...) en este caso, ha de resaltarse, además, la peculiaridad de que el decreto de prórroga constituye una formalización ad extra de la previa autorización del Congreso de los Diputados, esto es, su contenido es el autorizado por la Cámara, a quien corresponde autorizar la prórroga del estado de alarma y fijar su alcance, condiciones y términos, bien haciendo suyos los propuestos por el Gobierno en la solicitud de prórroga, bien estableciéndolos directamente. Al predicarse del acto de autorización parlamentaria, como ya se ha dejado constancia, la condición de decisión con rango o valor de ley (ATC 7/2012, FJ 4), idéntica condición ha de postularse, pese a la forma que reviste, de la decisión gubernamental de prórroga, que meramente se limita a formalizar y exteriorizar el acto parlamentario de autorización» (STC 83/2016, de 28 de abril, FJ 10).

22 Ambos Reales Decretos, a pesar de esta forma jurídica, quedarán sometidos, en razón de su condición de actos y disposiciones con fuerza o rango de ley, a un mismo régimen de control jurisdiccional ante el Tribunal Constitucional (STC 83/2016, de 28 de abril, FJ 11), que, en su calidad de «Guardián de la Constitución», lo ejercerá de manera monopolística. Sobre esta cuestión, véase Álvarez García, V., Arias Aparicio, F., y Hernández Diez, E., Lecciones jurídicas para la lucha contra una epidemia, Iustel, 2020, pp. 89 y ss.

23 Véanse, a este respecto, los arts. 11 y 12 LOAES. 
ejemplo, el art. 55.1 CE permite que, en el supuesto de la declaración del estado de excepción, el Gobierno de la Nación, previa autorización del Congreso de los Diputados, pueda suspender diversos derechos y libertades fundamentales (algo impensable en tiempos de normalidad). A saber: el derecho a la libertad personal (art. $17 \mathrm{CE}$, excepto su apartado 3); el derecho a la inviolabilidad del domicilio (art. 18.2); el derecho al secreto de las comunicaciones (art. 18.3 CE); la libertad de residencia y de circulación por el territorio nacional (art. 19 CE); la libertad de expresión [art. 20.1 a) CE]; el derecho a la información [art. 20.1 d) CE]; la prohibición del secuestro de publicaciones, grabaciones y otros medios de información (art. 20.5 CE); los derechos de reunión y de manifestación (art. 21 CE); el derecho de huelga (art. 28.2 CE); y el derecho a la adopción de medidas de conflicto colectivo (art. 37.2 CE).

\section{Los límites de los poderes de necesidad}

La necesidad es un concepto fundamental en el Derecho público. Tan fundamental como peligroso. En efecto, la necesidad, tal y como enseña la historia, ha sido invocada en numerosas ocasiones para justificar los más aberrantes abusos; tanto mayores, cuando menor ha sido el grado de desarrollo o de implantación del Derecho en un determinado Estado, y mayor la importancia del fin puesto en peligro y la gravedad y la inminencia de este peligro. Para evitar (o reducir al menos) los riesgos de abuso en la invocación de la necesidad es imprescindible establecer dos tipos de contrapesos. Por un lado, es necesario perfilar con rigor sus límites jurídicos (cosa en la que me centraré en este epígrafe) y, por otro, es fundamental establecer un sistema eficaz de control de la utilización de la necesidad (tema que abordaré en el apartado final de este trabajo).

Existen básicamente dos categorías de límites jurídicos frente al concepto de necesidad:

A) La primera se refiere a la efectiva existencia de una «situación de necesidad» $\mathrm{o}$, lo que es lo mismo, a la concurrencia real y simultánea del elemento fáctico y del teleológico. Cuando no exista una efectiva situación fáctica que haga peligrar la realización de un fin esencial, los Poderes Públicos no podrán invocar el concepto de necesidad para justificar sus actuaciones. La apreciación de la existencia y del contenido concreto tanto del presupuesto fáctico como del teleológico está atribuida, en primera instancia, a los Poderes Públicos en el momento de adoptar y de aplicar la medida. Pero, aunque cuentan para ello con un importante margen de valoración, sus apreciaciones serán perfectamente fiscalizables, en segunda instancia, por los órganos de control.

B) La segunda categoría de límites, que opera cuando efectivamente existe una situación de necesidad, sirve para perfilar el contenido concreto que 
puede tener cada una de las específicas medidas de necesidad o, lo que es lo mismo, para delimitar el alcance derogatorio y el adaptador que en un determinado supuesto puede revestir la necesidad. Todos los límites que integran esta categoría se reconducen al principio de proporcionalidad considerado en un sentido amplio, tal y como fue configurado hace ya muchísimos años por el Derecho de policía prusiano. La función última de este principio consiste en conciliar intereses contrapuestos, buscando un punto de equilibrio específico para cada conflicto, punto de equilibrio que encuentra su reflejo en cada medida de necesidad.

El concepto de proporcionalidad en sentido amplio es un concepto complejo integrado a su vez por tres (sub)principios de aplicación progresiva: en primer lugar, el de adecuación, idoneidad o congruencia; en segundo lugar, el de intervención minima o intervención menos lesiva; y, en tercer lugar, el principio de proporcionalidad en sentido estricto ${ }^{24}$.

a) El principio de adecuación, formulado de forma negativa, proscribe toda medida que no sirva para conectar efectivamente los dos términos del binomio fáctico-teleológico o, en otros términos, por no ir encaminada a establecer una superación exitosa de la situación de concreto peligro que amenaza a un determinado fin.

$24 \mathrm{El}$ art. 1.2 LOAES se refiere al necesario respeto del principio de proporcionalidad durante la aplicación de los estados constitucionales de emergencia. Dice este precepto que: «Las medidas a adoptar en los estados de alarma, excepción y sitio, así como la duración de los mismos, serán en cualquier caso las estrictamente indispensables para asegurar el restablecimiento de la normalidad. Su aplicación se realizará de forma proporcionada a las circunstancias».

Aunque es cierto que este precepto no incluye de manera expresa los tres escalones del principio de proporcionalidad recogidos en este trabajo, no es menos cierto que, a partir de la tercera prórroga del estado de alarma decretado el 14 de marzo de 2020, el Gobierno de la Nación justifica, sucesivamente, que las medidas de necesidad que adopta en cada uno de los Reales Decretos de prórroga son adecuadas para alcanzar el fin de luchar contra la pandemia, son las estrictamente indispensables y sus «beneficios son superiores a los perjuicios que producen sobre otros bienes jurídicos en conflicto». A este respecto, puede verse esta justificación del respeto del principio de proporcionalidad en sentido amplio en los preámbulos del RD 492/2020, de 24 de abril (tercera prórroga), del RD 514/2020, de 8 de mayo (cuarta prórroga), del RD 537/2020, de 22 de mayo (quinta prórroga) y del RD 555/2020, de 5 de junio (sexta prórroga). El Gobierno de la Nación abandonó, no obstante, esta sana práctica de justificar el respeto del principio de proporcionalidad con la finalización del primer estado de alarma, acudiendo a fórmulas extraordinariamente genéricas en el RD 900/2020, de 9 de octubre (declaración del estado de alarma para varios municipios de la Comunidad de Madrid), y en el Real Decreto 926/2020, de 25 de octubre (nuevo estado de alarma en todo el país para hacer frente a la segunda ola de la COVID-19). Y es que estos Reales Decretos no se molestan lo más mínimo en justificar ni la necesidad ni la proporcionalidad de las medidas de necesidad contenidas en sus textos, sino que acuden a sendos párrafos breves y estereotipados impropios de normas llamadas a afectar a los derechos fundamentales de millones de españoles. Fijándonos en el Decreto gubernamental de 25 de octubre, su preámbulo, en un texto muy similar al del 9 de octubre, dice únicamente que: «Para hacer frente a esta situación, grave y excepcional, es indispensable proceder a la declaración del estado de alarma. Las medidas que se contienen en el presente Real Decreto son las imprescindibles para hacer frente a la situación, resultan proporcionadas a la extrema gravedad de la misma y no suponen la suspensión de ningún derecho fundamental, tal y como prevé el artículo 55 de la Constitución». 
El principio de adecuación presenta una triple vertiente: la espacial (que impide que pueda invocarse válidamente la necesidad fuera del ámbito territorial en el que se verifica efectivamente la situación de peligro), la material (que impide la adopción de medidas de necesidad que, por exceso o por defecto, no permitan la superación del peligro) y la temporal (que proscribe todas aquellas medidas que se dicten, ya sea en un momento anterior a la aparición de cualquier situación de peligro, o ya sea en un momento posterior, es decir, una vez que ha desaparecido dicha situación por alteración bien del elemento fáctico o bien del teleológico $)^{25}$.

b) El principio de intervención mínima exige que, entre las diversas medidas adecuadas para la realización del fin, los Poderes Públicos elijan la menos lesiva para los intereses de los particulares y de la comunidad.

c) El principio de proporcionalidad en sentido estricto impone, por último, que la eventual lesión de los derechos e intereses particulares y generales provocada por la medida adecuada y menos lesiva no se encuentre «manifiestamente fuera de proporción» con respecto a los efectos beneficiosos derivados de la efectiva realización del fin, superando el peligro que le acechaba.

Sólo estarán justificadas por la necesidad, en conclusión, aquellas medidas que, dirigidas efectivamente a la superación de un peligro que amenace a un fin comunitario esencial, sean adecuadas para ello, lo menos lesivas posible y cuyos efectos negativos no se encuentren, además, manifiestamente fuera de proporción en relación con los efectos beneficiosos derivados de la realización del fin.

\section{Los controles de los poderes de necesidad}

Sería completamente inútil trazar unos límites precisos al concepto de necesidad si luego no existiesen unos órganos de control (tan vinculados por dichos límites como los propios Poderes Públicos titulares de los poderes de necesidad) con una capacidad suficiente para hacerlos respetar, evitando, o al menos reduciendo sensiblemente, los riesgos de abuso en su utilización.

25 La temporalidad es un elemento connatural al concepto de necesidad. El principio de la adecuación temporal del Derecho de necesidad, en efecto, tan sólo permite aquellas medidas extraordinarias adoptadas estrictamente durante la vigencia de la crisis, proscribiendo todas aquellas acordadas fuera de la franja de tiempo que va desde que nace la situación de peligro para el fin comunitario esencial (en el caso de la pandemia, la protección de la vida y de la salud de la población) hasta que desaparece. En otros términos, la necesidad debe estar presente durante toda la vida de la medida, desde su adopción hasta su ejecución efectiva. En el momento en el que desaparezca la situación de peligro a la que se debe hacer frente (que es la crisis de salud pública provocada por la COVID-19), dicha medida dejará de estar justificada por la necesidad y, a partir de ese instante, su ejecución sería ilícita. Sobre esta cuestión, véase Álvarez García, V., 2020, el año de la pandemia de la COVID-19, Iustel, 2021, pp. 146 y 147. 
El control de la necesidad debe ser encomendado a una pluralidad de órganos de diversa naturaleza. Con ello se posibilita, en primer lugar, la extensión efectiva del control a todos los intersticios del concepto de necesidad, cualquiera que sea la naturaleza y el rango de la medida en que ésta encuentre su expresión. La distinta naturaleza de los órganos de control hace, en segundo lugar, que se enfrenten a la fiscalización de las medidas desde ópticas muy distintas. La multiplicidad de órganos de control, en tercer lugar, dificulta el que puedan hacerse realidad los potenciales intentos de los Poderes de necesidad de dominar o de acallar todas las reacciones contra los posibles abusos en la invocación injustificada de la necesidad.

En este contexto, el control de la necesidad se efectuará a través de tres tipos de órganos que se valen de parámetros o criterios distintos para el desempeño de su tarea fiscalizadora. En primer lugar, están los órganos políticos de control, entre los que destaca fundamentalmente el Parlamento, que utiliza en su función de control parámetros de oportunidad (política) ${ }^{26}$; el segundo lugar, lo ocupan los órganos de naturaleza administrativa que utilizan en el desempeño de su tarea fiscalizadora parámetros de legalidad (juridicidad), junto a los estrictamente de oportunidad (político-administrativa ${ }^{27}$ ); y, en tercer lugar, y como mecanismo de cierre, se encuentran los órganos jurisdiccionales, que son los que tienen encomendado en última instancia el control, basado en parámetros exclusivamente jurídicos, sobre la existencia o no de la necesidad justificadora de la medida, aun cuando ésta haya pasado previamente de manera satisfactoria los oportunos controles políticos o/y administrativos ${ }^{28}$.

26 El desencadenamiento de la crisis de salud pública originada por la COVID-19 ha provocado, como ya sabemos, la activación del estado constitucional de alarma en varias ocasiones desde el 14 de marzo de 2020. La activación de este estado excepcional, sin necesidad de ir más lejos, nos ofrece sendos ejemplos de dos tipos distintos de controles parlamentarios: A) El estado de alarma lo declara el Gobierno de la Nación. Una vez declarado, este órgano constitucional debe informar al Congreso de los Diputados. Esto es, la Cámara Baja realiza un control político estrictamente a posteriori (art. 116.2 CE, art. 162 RCD y art. 8.1 LOAES). B) Algo diferente ocurre con la prórroga del estado de alarma (o la declaración y prórroga del estado de excepción). Aunque esta prórroga es competencia gubernamental, requiere, sin embargo, de la autorización previa del Congreso de los Diputados. Esto es, sin la intervención previa de la Cámara Baja, no resulta posible la activación gubernamental del estado de alarma (art. 116.2 CE, art. 162 RCD y art. 6.2 LOAES).

27 Un ejemplo paradigmático de este tipo de control es el ejercido por el Consejo de Estado. Téngase en cuenta, en todo caso, que mediante el control de legalidad se verifica si las medidas de necesidad respetan el conjunto del ordenamiento jurídico (que es de obligado cumplimiento para todos, también para los poderes públicos); mientras que la fiscalización en base a criterios de oportunidad permite controlar suplementariamente si las medidas que pretenden aplicarse (y que son plenamente legales) resultan, además, razonablemente buenas y convenientes para la consecución del fin de interés general perseguido con su adopción.

28 Siguiendo con el ejemplo ofrecido por el estado de alarma en España, nuestro Tribunal Constitucional ejerce monopolísticamente el control sobre los Reales Decretos dictados por el Gobierno para su declaración y para su prórroga, dado que este Alto Tribunal ha considerado en sus pronunciamientos dictados a raíz de la «crisis de los controladores aéreos» de diciembre de 2010 que dichos Reales Decretos (a pesar de su forma jurídica) tienen valor de ley (ATC 7/2012, de 13 de enero; y STC 83/2016, de 28 de abril).Fuera de estos casos, los actos y disposiciones de las Administraciones Públicas adoptados durante la vigencia de los estados constitucionales de excepción podrán ser impugnados ante la jurisdicción ordinaria (art. 3.1 LOAES). Otro ejemplo de control jurídico por parte de los Jueces y Tribunales ordinarios, muy de moda durante los 
TITLE: The foundations of Law of the exception in times of Covid-19

ABSTRACT: The author, in these times of the coronavirus pandemic, reviews, firstly, the historical models of the Law of the exception in the Western world; and secondly, it studies the components that should order this Law of crisis today. In this context, this work, starting from the idea that the theory of emergency does not constrain its virtuality only to the most extreme dangers for the life of States, recalls the obligation of the Public Powers to ensure the realization of the essential purposes of the social community, as well as the shaping elements of the concept of emergency, its fundamental effects, its limits and its controls.

RESUMEN: El autor, en estos tiempos de la pandemia de coronavirus, hace un repaso, en primer término, de los modelos históricos del Derecho de necesidad en el mundo occidental, para pasar, en un segundo término, al estudio de los componentes que deben ordenar este Derecho de las crisis en la actualidad. En este contexto, este trabajo, partiendo de la idea de que la teoría de la necesidad no constriñe su virtualidad únicamente a los peligros más extremos para la vida de los Estados, recuerda la obligación que asiste a los Poderes Públicos de asegurar la realización de los fines esenciales de la comunidad social, así como los elementos configuradores del concepto de necesidad, sus efectos fundamentales, sus limites y sus controles.

KeY words: Public Law, Law of the exception, Law of crisis, states of emergency, emergency powers, exceptional circumstances, epidemics, COVID-19.

Palabras clave: Derecho público, derecho de necesidad, derecho de crisis, estados de emergencia, poderes de necesidad, circunstancias excepcionales, epidemias, COVID-19.

FECHA DE RECEPCIÓN: 02.03.2021

FECHA DE ACEPTACIÓN: 20.09.2021

últimos meses de pandemia, es el relativo a la obligación de autorización o de ratificación por los Jueces y Tribunales de lo Contencioso-Administrativo de las medidas sanitarias que impliquen una restricción de derechos fundamentales, como la que supone, por ejemplo, el confinamiento de poblaciones [arts. 8.6, 10.8 y 11.1 i) LJCA]. 